\title{
EDITORIAL
}

\section{To all involved - we have a problem}

\section{We do not have clear information ... about the costs ... from the manufacturer to the doctor's office}

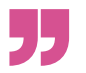

Tempting as it is to adopt a simplistic well-meaning approach, we don't consider that merely publishing articles on the financial toxicity of cancer constitutes an achievement for Nature Reviews Clinical Oncology as a journal for the medical oncology community. But we regularly feature articles on the cost of anticancer treatments and have been doing so for more than a decade. What made us consider those articles appropriate for our journal is the proactive approach of their authors, which we hope our readers have also found inspiring. In a nutshell: we publish discussions of potential solutions and avoid 'moaning exercises' (empty descriptions of the current bleak panorama).

Two perfect examples of the type of article we consider of interest for our readers are featured in the July 2018 issue of the journal. In a provocative Comment, Vinay Prasad, Christopher McCabe and Sham Mailankody discuss a "thought experiment" (REF. $\left.{ }^{1}\right)$ in which they evaluate whether pharmaceutical companies could derive profits from conducting clinical trials with inert anticancer therapies. Their conclusion is sobering: any anticancer drug that generates a US $\$ 440$ million profit and is approved on the basis of the results of a single clinical trial would justify a hypothetical portfolio involving 100 inert compounds. This scenario is an exaggerated distortion of reality, and the authors "certainly do not believe that companies are actively pursuing ineffective drugs", but the revenue they propose actually matches those of many agents currently used in clinical practice. Let's not forget that anticancer agents remain the best-selling drugs among FDAapproved therapies (32\% of sales projected in 2017$)^{2}$. Another fact to keep in mind is that many drug approvals are indeed based on the results of a single trial, which do not always meet the threshold for meaningful clinical benefit ${ }^{3}$. Mailankody et al. state that the riskbenefit balance in oncology clinical trials (regardless of whether they are intended to lead to drug approval) remains to be properly addressed. They do not explicitly formulate a request but, after reading their article, we cannot help but ask for transparency from the regulatory bodies regarding the criteria they use for drug approvals.

Apropos of transparency, Carin Uyl-de Groot and Bob Löwenberg believe that this is the principle that should guide the pricing of anticancer agents. In their News \& Views article ${ }^{4}$ they present an algorithm that combines the key factors that should be considered to set the price of a drug: $R \& D$ costs, market-related costs, patent duration, target patient population and profit margin. Of note, in their model, profit is proportionally related to clinical benefit. These authors directly address the role of regulators who, in their opinion, should actively guarantee that anticancer agents are fairly priced and promote access to them.

Clinical benefit is a common theme in these two articles. 'Value' and 'benefit' can mean different things, even in the context of patient management. To avoid ambiguity, a few years ago several groups of experts independently established frameworks to define clinical benefit through a series of set criteria. Importantly, this commendable effort was led by the two major medical oncology societies, ESMO and ASCO. As proposed, we consider that regulatory bodies should ensure transparency in drug approvals and in the subsequent pricing of such drugs. Perhaps only societies with the reputation of ESMO and ASCO will be able to reach out to the regulatory bodies and ask them to bridge the gap between what their frameworks define as meaningful clinical benefit and the outcomes derived from treatment with most of the innovative agents currently available.

Both articles have been written by professionals from an academic environment and located in highincome countries. As editors of Nature Reviews Clinical Oncology, we will keep publishing articles about value if, by doing so, we can foster real discussion. We are aware that we need to include all the voices that belong in this debate and have a constructive opinion. We are interested, in particular, in hearing from professionals in the pharmaceutical industry. We understand that drug development needs to be profitable to those who invest an effort in such a complex task. We do not have clear information, however, about the costs associated with the different stages of marketing of a therapeutic agent, from the manufacturer to the doctor's office.

We have a problem: the rising cost of anticancer therapies and the current regulatory environment have helped to create an unsustainable (and unacceptable) situation. This problem frequently receives attention in our journal - and we consider that our duty is to do so. This is a call to all stakeholders to state what their position is in face of this problem. We, as editors, are committed to open the communication channel to everyone with a meaningful contribution. Changes will not happen quickly, but they need to happen.

1. Prasad, V., McCabe, C. \& Mailankody, S. Nat. Rev. Clin. Oncol. https://doi.org/10.1038/s41571-018-0030-2 (2018). Baedeker, M. et al. Nat. Rev. Drug Discov. 17, 87 (2018).

3. Booth, C. M. \& Del Paggio, J. C. Nat. Rev. Clin. Oncol. 14, 135-136 (2017).

4. Uyl-de Groot, C. A. \& Löwenberg, B. Nat. Rev. Clin. Oncol. https://doi.org/10.1038/s41571-018-0027-x (2018). 